\title{
OTIOTOMICS
}

Revista de economía, empresa y sociedad

Dossier «Economía social y solidaria: experiencias y retos»

\section{La financiación del emprendimiento social, ¿modelos nuevos para problemas viejos?}

Flisabet Bach

Profesora colaboradora de los Estudios de Economía y Empresa (UOC)

\section{Laura Lamolla}

Profesora de los Estudios de Economía y Empresa (UOC)

Agradecimientos por su colaboración a:

Valentí Acconcia, de Vanacco, y Amadeo Ibarz y Montse Tarridas, de Think Great

RESUMEN En la medida en que siempre han habido problemas sociales a los que dar respuesta y soluciones, ha existido emprendimiento social. En los últimos años se han desarrollado nuevas formas empresariales que ofrecen una respuesta a retos sociales y medioambientales que combinan aspectos de las entidades no lucrativas con otros de las empresas lucrativas. Por un lado, en este artículo definimos el emprendimiento social, en el sentido de que tiene unas características y unas necesidades específicas y diferenciadas del emprendimiento convencional. Ello ha permitido el desarrollo de un mercado de financiación específico que intenta dar una respuesta adecuada a las distintas tipologías de entidades que operan en este campo. Y por el otro, presentamos los modelos de financiación para iniciativas sociales y en qué casos y situaciones son más adecuados unos que otros.

PALABRAS CLAVE emprendimiento social; entidades no lucrativas; modelos de financiación

The financing of social entrepreneurship. New models for old problems?

ABSTRACT Social entrepreneurship has existed for as long as there have been social problems needing responses and solutions. In recent years, new forms of business have been developed that respond to social and environmental challenges, and which combine aspects of non-profit organizations with others of for-profit companies.

In this article we start by defining social entrepreneurship, which has specific characteristics and needs that are distinct from those of conventional entrepreneurship. This difference has enabled the development of a financing market specific to this field, which attempts to provide suitable responses to the different types of organizations operating therein.

We then go on to look at the financing models available to social initiatives and the cases and situations that make some more appropriate than others.

KEYWORDS social entrepreneurship; non-profit organizations; financing models 


\section{Introducción}

El emprendimiento social ha existido siempre y en todo el mundo, y actualmente representa el $3,2 \%$ del total de empresas en su fase inicial (Bosma y otros, 2016). Dado que ni los estados ni el mercado satisfacen plenamente las necesidades sociales de las personas, a lo largo de la historia han surgido emprendedores con la intención de dar respuesta a diversas necesidades, generalmente básicas, como el acceso a la salud y a la educación, pero también otras como el acceso a las nuevas tecnologías o el ocio infantil.

En primer lugar, está ampliamente aceptado que los estados han fallado y fallan a la hora de distribuir los recursos, especialmente entre la población más pobre, que tiene dificultades de acceso e inferior calidad en los servicios, entre otras cuestiones. En segundo lugar, y en relación con el mercado, muchos consumidores, especialmente de países en vías de desarrollo, no pueden pagar los productos y los servicios que podrían satisfacer sus necesidades. De hecho, las empresas han tenido pocos incentivos a la hora de dirigirse a estos mercados con muy bajo poder adquisitivo y de capacidad de consumo, aunque en la última década han aparecido nuevos modelos de negocio, como los llamados «de la base de la pirámide» (BdP) -modelos que desarrollan, gracias a la innovación, productos y servicios dirigidos a los millones de personas que se encuentran en las capas más pobres de la sociedad, es decir, que se encuentran «en la base de la pirámide»-, en los que precisamente este segmento de mercado forma parte de la cadena de valor de la empresa, ya sea como cliente, proveedor, etc.

La forma tradicional de abordar los problemas sociales se ha basado en el modelo de las subvenciones por parte de la Administración y de donaciones altruistas que han financiado las actividades y a las entidades de índole social. Esta situación genera dependencia de terceros e inestabilidad en los ingresos de las entidades sociales. No obstante, en los últimos años se han desarrollado modelos de negocio que combinan características de los sectores lucrativo y no lucrativo. Son las llamadas "empresas híbridas", es decir, que combinan un interés social (modelo de entidades no lucrativas) con la financiación a partir del beneficio generado por la propia organización y con recursos ajenos, que también reciben una remuneración por esta financiación (modelo lucrativo). Estos nuevos modelos permiten afrontar problemas sociales con respuestas viables y sostenibles económicamente. A su vez, en el ámbito de la financiación también han surgido nuevos modelos que permiten dar respuesta tanto a las necesidades financieras de estas empresas híbridas, como a las de las entidades y personas que las financian.

En este artículo analizamos las diversas alternativas de financiación que actualmente tienen las empresas sociales, con especial atención a las de nueva creación. Antes haremos un análisis de lo que significa el emprendimiento social para consensuar posiciones.

\section{Una aproximación al emprendimiento social}

Como en el convencional, el emprendimiento social detecta las necesidades y actúa allí donde el mercado y las instituciones públicas no llegan. Es decir, detecta oportunidades para mejorar productos y servicios, crear soluciones y nuevas aproximaciones en el ámbito social.

De todas maneras, todavía no existe un consenso absoluto para definir el emprendimiento social, pese a haber pasado ya muchos años desde que Bornstein (2004) escribió How to Change the World. Social Entrepreneurs and the Power of New ldeas, uno de las principales obras de referencia en este ámbito, en la que hacía una recopilación de casos de emprendimiento social, identificando las características de los protagonistas y las actividades que llevaban a cabo.

Por un lado, el emprendimiento social tiene puntos de encuentro con diversas disciplinas de las ciencias sociales (economía, ciencias políticas, management, sociología, etc.) que tienen diversos enfoques sobre el mismo fenómeno. Y por otro, la investigación se ha centrado en una o diversas dimensiones del fenómeno, como son los individuos (características sociodemográficas, valores, actitudes, etc.), las actividades en que se focaliza (reducción de la pobreza, acceso a la salud y a la educación, conservación de ecosistemas, etc.), los procesos que la caracterizan, los recursos que moviliza y la misión. De hecho, algunos autores, como Dacin y otros (2011), 
consideran que lo que realmente distingue el emprendimiento social de otras iniciativas emprendedoras es precisamente la misión.

El emprendimiento social contempla en su misión (la razón de ser de la entidad) la creación de valor social y, aunque algunos autores ignoran en su definición los resultados económicos que de ello se derivan, otros consideran que son una parte fundamental de la misma. No obstante, unos la consideran en un nivel secundario (Mair y Martí, 2006; Seelos y Mair, 2005) y otros (Dacin y otros, 2011) en el mismo nivel. Como indican estos últimos, el valor económico es imprescindible para la sostenibilidad de las iniciativas sociales. Si no fuera así, no se trataría de emprendimiento social, sino de otras iniciativas de carácter social, como la filantropía o incluso la actividad de la Administración pública. Bosma y otros (2016) utilizan en su definición ambas aproximaciones. En un sentido amplio, definen el emprendimiento social como cualquier actividad, organización o iniciativa que tiene un objetivo concreto relacionado con aspectos sociales y/o medioambientales. Y de un modo más acotado, la misión y el valor generado deben tener claramente un objetivo social o medioambiental.

\section{Tabla 1. Principales diferencias entre emprendimiento social (ES) y convencional (EC)}

\begin{tabular}{l|l|l}
\hline \multicolumn{1}{|c|}{ MS } & \multicolumn{1}{|c|}{ ES } & \multicolumn{1}{c|}{ EC } \\
\hline Misión & Necesidad social no atendida & $\begin{array}{l}\text { Detección de una oportunidad de mercado } \\
\text { que permite captar valor económico (para } \\
\text { la empresa) }\end{array}$ \\
\hline Valor & Valor social como finalidad principal & Valor económico como finalidad principal \\
\hline
\end{tabular}

Fuente: Elaboración propia.

Como se puede observar en la tabla anterior, el tipo de valor para ambas tipologías de emprendimientos no es excluyente, a pesar de que la finalidad principal sea una $u$ otra. De hecho, esta doble vertiente permite definir un universo amplio de entidades y empresas que participan del ámbito social, pero desde distintos focos y perspectivas.

Figura 1. Clasificación del emprendimiento social en función del impacto social y la orientación al mercado

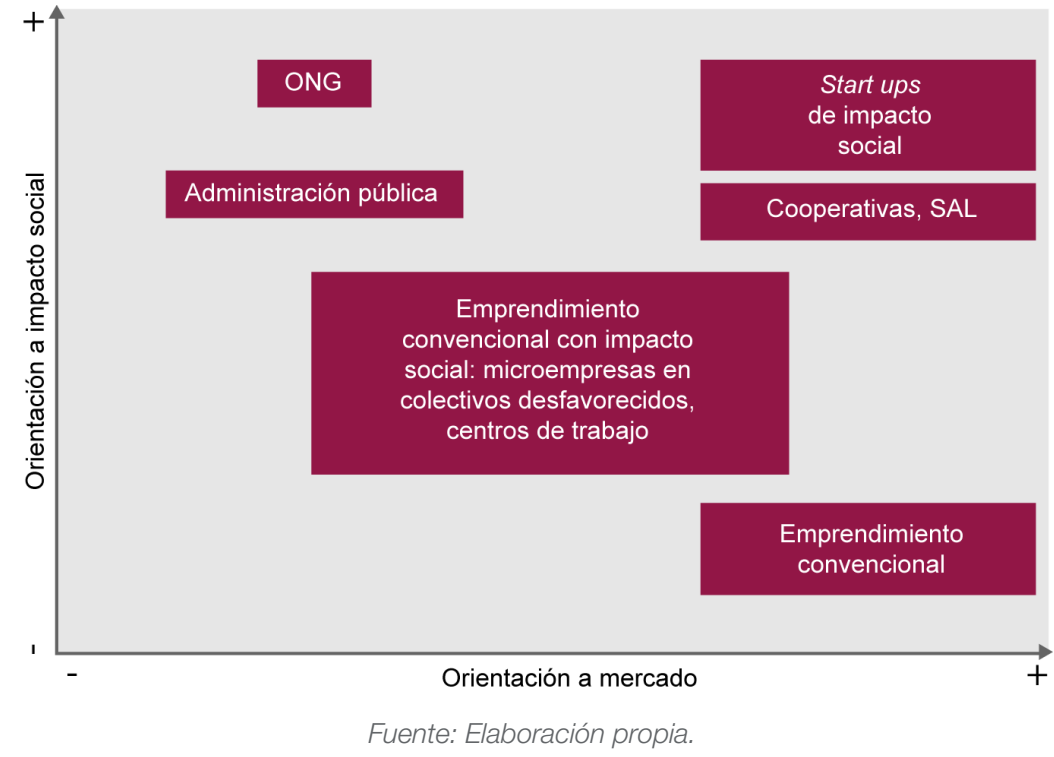

Como podemos observar en el gráfico anterior, las ONG y los ámbitos de actuación de la Administración pública se sitúan donde el impacto social es más elevado y la orientación al mercado es baja. 
A continuación, con un impacto social elevado y con cierta orientación al mercado, se encuentran esas entidades y empresas cuya actividad comercial es subsidiaria de su misión. En este apartado podemos encontrar, por ejemplo, centros de trabajo para colectivos con discapacidades físicas o psíquicas o entidades que ayudan a personas de colectivos desfavorecidos a montar su propia microempresa, entre otros.

Seguidamente, con una mayor orientación al mercado pero también manteniendo un impacto social, se sitúa el sector del cooperativismo, donde la gestión de las entidades es participativa por parte de los trabajadores. Este elemento aporta un aspecto social esencial a esta tipología de entidades, más allá de que su misión sea de finalidad social o comercial.

Entrando ya en las empresas con una clara orientación al mercado pero que mantienen una vocación social elevada, se sitúan las empresas de impacto social o empresas híbridas. Dentro de este colectivo se incluyen las startups de impacto, es decir, aquellas empresas de nueva creación con un elevado potencial de crecimiento, alto rendimiento económico y una elevada incidencia social.

Finalmente, con una clara vocación hacia el mercado se sitúan las empresas comerciales. En este caso, el impacto social, más allá del propio de cualquier empresa, dependerá también de cómo ejercite su responsabilidad corporativa. Hay empresas que son muy activas en este aspecto y generan una incidencia social elevada y otras que no.

Así pues, teniendo en consideración esta clasificación, y en base a la definición de emprendimiento social más restrictiva, que contempla tanto la orientación al mercado como el impacto social, se puede afirmar que las ONG, la Administración pública y el emprendimiento convencional -a pesar de realizar acciones a través de la responsabilidad social corporativa-, quedan excluidos del emprendimiento social.

\section{Financiación de iniciativas de emprendimiento social}

En los últimos años han surgido nuevos modelos que, para que el proyecto sea viable y autosostenible, combinan la misión social con la rentabilidad económica. Este nuevo modelo amplía las tradicionales formas de financiación basadas en las donaciones altruistas y las subvenciones de los organismos públicos hacia fórmulas relacionadas con el modelo de empresa convencional.

Figura 2. Clasificación de las formas de financiación según el grado de impacto social y orientación al mercado de la iniciativa financiada

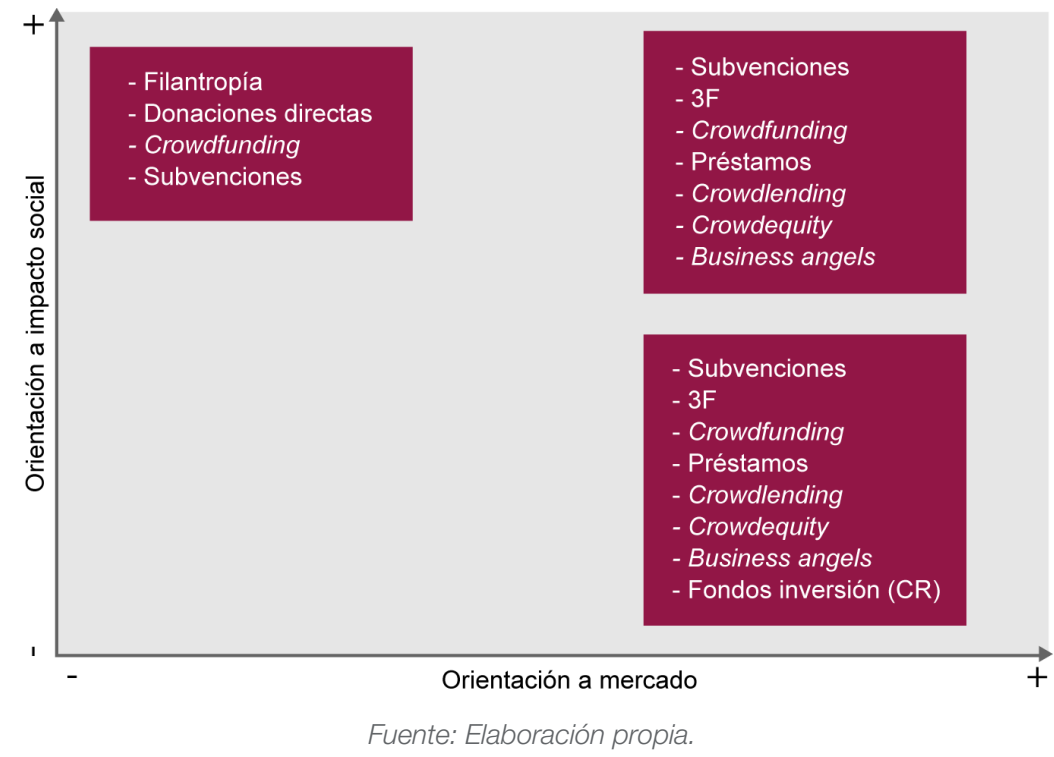


En este análisis nos centraremos en las formas de financiación orientadas a lo que hemos definido como emprendimiento social en el apartado anterior. Como se puede observar en la figura anterior, las herramientas de financiación son similares a las que acceden las empresas convencionales. En relación con las entidades que ceden los fondos, en algunos casos también pueden coincidir con las que operan en el ámbito de las empresas comerciales, pero en otros hay iniciativas especializadas en emprendimiento social, ya sea por su idiosincracia o por las formas jurídicas que financian (fundaciones, centros especiales de trabajo, cooperativas, sociedades anónimas laborales, etc.). No pretendemos hacer una relación exhaustiva, sino explicar la tipología de cada forma de financiación y poner ejemplos de referencia:

Las aportaciones propias de los promotores del proyecto y de su entorno inmediato: Este tipo de financiación hace referencia a las aportaciones que los mismos promotores o bien su círculo familiar, de amigos y conocidos hacen en las primeras etapas del proyecto para que se puede realizar un estudio/investigación de mercado, desarrollar el producto y cubrir los gastos iniciales.

Las donaciones y aportaciones a fondo perdido: en este caso, el proyecto recibe recursos -no necesariamente económicos- de terceros, pero sin la obligación de devolverlos al donante. En este grupo hay entidades/instituciones que tradicionalmente han participado en la financiación de empresas y proyectos, como la Administración pública. A modo de ejemplo, se pueden mencionar SME Instruments -dirigida a empresas con potencial de dar respuesta a problemas europeos, también en el ámbito social, económico y medioambiental-, y las subvenciones del Departamento de Empresa y Ocupación para la contratación de avales para la constitución de cooperativas y sociedades anónimas laborales. Las fundaciones también han realizado tradicionalmente donaciones en este ámbito, aunque en la actualidad las hay con la finalidad específica de promover y apoyar el emprendimiento social, como la Fundación Repsol para proyectos de mejora del impacto medioambiental.

Hay otras fórmulas más recientes y que constituyen por sí mismas modelos de negocio innovadores, como el crowdfunding, una forma de financiación colectiva organizada a través de plataformas que operan en línea. Hay tres tipos de crowdfunding a fondo perdido:

- Crowdfunding de donación: Es una fórmula muy habitual en ONG y muy poco en empresas, pero es posible aplicarla cuando una ONG pide los fondos con el fin de que el colectivo al que se dirige pueda desarrollar proyectos de emprendimiento. También se puede utilizar si una empresa, en este caso social, quiere llevar a cabo un proyecto de tipo altruista.

- Crowdfunding de recompensa, que queda fijada previamente y depende de la cantidad aportada. Este tipo de crowdfunding, por ejemplo Goteo, también aporta información a los promotores-donantes sobre su producto, su aceptación y la tipología de sus clientes. Aparte de obtener financiación, es una forma de validar el proyecto.

- Crowdfunding mixto de donación y recompensa: Es una fórmula en que la recompensa no la da la entidad que recibe los fondos, sino otra entidad de renombre, cuyo producto puede ser interesante para las personas que aportan los fondos. Como en el caso del crowdfunding de donación, es poco habitual en el ámbito de proyectos empresariales.

Las aportaciones de capital y préstamos participativos son un tipo de financiación en el que las personas/ entidades que aportan los fondos reciben a cambio participaciones de la sociedad receptora. Respecto a las aportaciones de capital hay de diversos tipos:

- En el crowdequity, o crowdfunding de capital, los fondos provienen, como en los casos del crowdfunding de donación y de recompensa, de diferentes personas que actúan mediante plataformas en línea, como por ejemplo La Bolsa Social.

- Los business angels especializados en proyectos de impacto social: Igual que el resto de business angels, son inversores privados organizados habitualmente en torno a redes o aceleradoras y que invierten directamente en los proyectos. Aparte de la aportación económica, y a diferencia del crowdequity, también contribuyen al éxito del proyecto con su experiencia y red de contactos. Hay múltiples redes, como Creas Coinversión (red de business angels dirigida a startups de impacto social) o Ship2b (aceleradora especializada en proyectos con impacto social). 
- El emprendimiento social también tiene acceso al capital riesgo, que comprende aquellas entidades que aportan fondos a las empresas en forma de capital por un tiempo determinado. Los fondos que aportan pueden estar por encima de los trescientos mil euros. Normalmente, estos fondos se especializan por sector/actividad de las empresas financiadas o por su etapa de desarrollo. Por ejemplo, Creas Inicia se dirige a startups en fase semilla, Creas Desarrolla a startups de impacto social en fase de expansión y la Fundación Isis está especialmente dirigida a la inclusión social y a centros de trabajo.

Finalmente, los préstamos participativos son una fórmula mixta entre las aportaciones de capital y los créditos, ya que son aportaciones que deben devolverse en un plazo acordado y generan un interés, aunque con un tramo que es variable en función del resultado de la empresa. Se debe puntualizar que este tipo de financiación se considera un recurso propio dentro del balance de la sociedad. La Administración pública (Enisa, Institut Català de Finances) dispone de modalidades de préstamos participativos, muchas veces complementarios a las inversiones de inversores privados, como los business angels. Algunas entidades sin ánimo de lucro tienen como finalidad la financiación de proyectos de economía social. Este es el caso de Coop57, cooperativa de servicios financieros que facilita préstamos participativos a las cooperativas adheridas.

Los préstamos son un tipo de financiación en la que la entidad que aporta los fondos y la que los recibe acuerdan un plazo para devolverlos y una remuneración (interés). Como en las tipologías anteriores, están los agentes tradicionales que han concedido préstamos pero se han especializado en el sector social y otras que han innovado en el modelo de negocio, como es el caso del crowdlending.

El crowdlending es la modalidad de préstamo del crowdfunding, es decir, los fondos provienen de personas privadas que los ceden a través de una plataforma en línea, que selecciona los proyectos y gestiona el buen fin de la operación hasta la devolución total del nominal y de los intereses. En el caso del emprendimiento social, el interés que se acuerda puede ser un interés de mercado o ser nulo, de modo que la devolución para el aportante sea el impacto social. A diferencia del emprendimiento comercial, en el que los préstamos se conceden a empresas que ya llevan tiempo en el mercado para poder contrastar su solvencia, en el caso del emprendimiento social el crowdlending también se utiliza para financiar proyectos nuevos, como son los casos de Ecrowd para proyectos de impacto medioambiental o Realfunding.

Hay algunas entidades financieras, tanto del sector de la banca ética (Fiare Banca Ética) como de la banca comercial tradicional (Microcréditos de Microbank), que tienen líneas de préstamo dirigidas exclusivamente a financiar proyectos de emprendimiento social.

Finalmente, algunas entidades no lucrativas (por ejemplo, ASCA facilita microcréditos sin interés dirigidos a personas en el paro) tienen líneas de microcréditos dirigidas a personas de colectivos con riesgo de exclusión para que puedan financiar sus proyectos. Las instituciones públicas -por ejemplo, el Institut Català de Financescuentan también con líneas de préstamo para iniciativas sociales.

Pese a toda esta diversidad de tipos de financiación, en Europa occidental las principales fuentes de financiación que utilizan los emprendedores sociales en etapas iniciales son, por orden de importancia, los programas gubernamentales y donaciones (43\%), la familia (38\%), bancos, otras instituciones financieras (25\%) e inversores privados y capital riesgo (25\%), jefes y compañeros de trabajo (18\%), amigos (14\%) y en último lugar el crowdfunding (9\%) (Bosma y otros, 2016). Esta situación parece reflejar que a pesar de que en los últimos años han surgido nuevos instrumentos de financiación, el emprendimiento social sigue financiándose principalmente a través de programas gubernamentales y de la familia y conocidos (las $3 \mathrm{~F}$ ).

\section{Conclusiones}

En la medida en que siempre han habido problemas sociales a los que dar respuesta y soluciones, ha existido emprendimiento social.

En los últimos años se ha intentado definir el emprendimiento social desde el ámbito académico. Existe un cierto consenso que se basa en dos elementos esenciales: la misión social y la captura de valor económico, este 
último necesario para la viabilidad y la sostenibilidad de la iniciativa. Es decir, necesario para dar respuesta a la necesidad social y/o medioambiental planteada en la misión de forma regular y sostenible en el tiempo.

Esta nueva forma de dar respuesta a retos sociales y medioambientales ha desarrollado nuevas formas empresariales que combinan aspectos de las entidades no lucrativas con otros de las empresas lucrativas. Es lo que llamamos «empresas híbridas».

Paralelamente, se está desarrollando un mercado de financiación que intenta dar respuesta a las distintas tipologías de entidades que operan en el campo del emprendimiento social.

Hay distintas tipologías de financiación según el retorno que se espera conseguir: a fondo perdido (si no se espera retorno), de participación en capital (si se espera un retorno variable y con un riesgo acorde con la evolución de la empresa) y de préstamo (si se espera una remuneración económica acordada).

También hay diferentes tipos de entidades que ofrecen la financiación: entidades sin ánimo de lucro, instituciones públicas y de iniciativa privada (personas físicas y personas jurídicas).

Otra diferencia es que cada entidad se dirige a una parte del universo que llamamos emprendimiento social: aquellas que se dirigen a las empresas híbridas y, muchas veces, concretamente a las startups de impacto; las que se han especializado en las empresas de tipo cooperativo, y las que dirigen sus fondos a aquellas empresas en las que la actividad comercial es subsidiaria de la misión social.

El emprendimiento social tiene una presencia muy dispar en los diferentes países del mundo; en el caso de España, posee una ratio que la sitúa en la zona baja (0,9\% según Bosma y otros, 2016). Es de esperar que, de acuerdo con lo que sucede en otras economías próximas, este sea un sector en crecimiento, tanto a nivel de proyectos como a nivel de formas, fuentes y volumen de fondos de financiación.

\section{Referencias bibliográficas}

BOSMA, N.; SCHOTT, T.; TERJESEN, S.; KEW, P. (2016). Global Entrepreneurship Monitor 2015 to 2016: Special Report on Social Entrepreneurship. Global Entrepreneurship Research Association (www.gemconsortium.org). BORNSTEIN, D. (2004). How to Change the World. Social Entrepreneurs and the Power of new Ideas. Oxford: Oxford University Press.

CARTER, E.; SHAW S. (2007). "Social Entrepreneurship». Social and small business and enterprise development. Vol. 14, n. ${ }^{\circ}$ 3, págs. 418-434.

COMISSIÓ EUROPEA. «The SME Instrument». Horizon 2020. Programa Marc per a la innovació i la recerca [artículo en línea: <https://ec.europa.eu/programmes/horizon2020/en/h2020-section/sme-instrument>]. [Fecha de consulta: 9 de octubre 2016].

DACIN, M. T.; DACIN, P.; TRACEY, P. (2011). «Social entrepreneurship: a critique and future directions». Organizations science. Vol. 22, n. 5, págs. 1203-1213.

FERNÁNDEZ-LAVIADA; PEÑA, I.; GUERRERO, M. I.; GONZÁLEZ-PERNÍA, J. L. (2015). Global Entrepreneurship Monitor. Informe GEM España 2014. Santander: Editorial de la Universidad de Cantabria.

GENERALITAT DE CATALUNYA. «Emprenedoria Social. Recull de recursos per a l'emprenedoria social» [en línea: $<$ http://www.emprenedoriasocial.cat/recull/index.php/recursos/listar/economics/589>]. [Fecha de consulta: 16 de octubre 2016].

MAIR, J.; MARTÍ, I. (2006). «Social entrepreneurship research: a source of explanation, prediction and delight». Journal of World Business. №. 41, págs. 36-44.

RUIZ DE MUNAIN, J. L.; MARTíN, J. (2012). Mapa de las inversiones de impacto en España. Madrid: Fundación Compromiso y Transparencia.

SEELOS, C.; MAIR, J. (2005). «Social entrepreneurship: creating new business models to serve the poor». Business Horizons. №. 48, págs. 241-246.

XARXANET.ORG. Entitats i voluntariat per un món millor: <http://xarxanet.org>. 


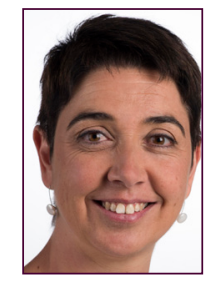

\section{Flisabet Bach Oller}

ebach@uoc.edu

Profesora colaboradora de los Estudios de Economía y Empresa (UOC)

Consultora económica y financiera de emprendedores en el proceso de creación y desarrollo del proyecto y de empresarios en la consolidación de la empresa y viabilidad de proyectos. Colegiada en el Colegio de Economistas de Cataluña y miembro del grupo de Trabajo de Creación de empresas y emprendimiento. Realiza charlas y cursos de formación sobre creación de empresas, finanzas y financiación de la empresa. Licenciada y máster en Administración y Dirección de Empresas por ESADE Business School. Colabora con la UOC como profesora colaboradora de la asignatura Iniciativa Emprendedora de los estudios de Economía y Empresa.

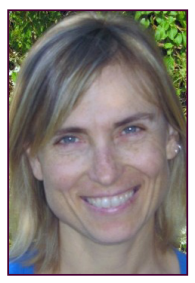

\section{Laura Lamolla} llamollak@uoc.edu Profesora de los Estudios de Economía y Empresa (UOC)

Profesora en la UOC desde 2007, donde imparte asignaturas de iniciativa emprendedora, estrategia y estrategia internacional. Combina su actividad docente con la dirección académica del grado en ADE. Sus intereses de investigación están relacionados con el género, la iniciativa emprendedora y el equilibrio vida-trabajo. Ha participado en diversos proyectos europeos y nacionales sobre estos temas. Doctora en Ciencias Políticas y de la Administración por la Universidad Autónoma de Barcelona, máster en International Management por CEMS (Community of European Management Schools) y licenciada y máster en Administración y Dirección de Empresas por ESADE Business School.

Los textos publicados en esta revista están sujetas -salvo que se indique el contrario- a una licencia de Reconocimiento 3.0 España de Creative Commons. Podéis copiarlos, distribuirlos, comunicarlos públicamente y hacer obras derivadas siempre que reconozcáis los créditos de las obras (autoría, nombre de la revista, institución editora) de la manera especificada por los autores o por la revista. La licencia completa se puede consultar en http://creativecommons.org/licenses/by/3.0/es/deed.ca.

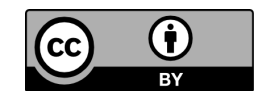

